\title{
Protecting persons at-risk of viral hepatitis and alcohol-related liver disease during the pandemic and beyond: mitigating the impact of coronavirus disease 2019 on liver health
}

\author{
Belaynew W Taye ${ }^{1}$ 옹 Patricia C Valery ${ }^{2}$, Paul J Clark ${ }^{3}$ (1) \\ 1 Department of Epidemiology, Bahir Dar University, Bahir Dar, Ethiopia; Faculty of Medicine, The University of Queensland, Brisbane, Australia; Mater \\ Research Institute, Brisbane, Australia, 2 Faculty of Medicine, The University of Queensland, Brisbane, Australia; Mater Research Institute, Brisbane, \\ Australia, ${ }^{3}$ Faculty of Medicine, The University of Queensland, Brisbane, Australia; Mater Research Institute, Brisbane, Australia; QIMR Berghofer \\ Medical Research Institute, Brisbane, Australia; Department of Gastroenterology and Hepatology, Mater Hospitals, Brisbane, Australia \\ Keywords: covid-19, harm reduction, viral hepatitis, alcohol-related liver disease \\ https://doi.org/10.29392/001c.24940
}

\section{Journal of Global Health Reports}

Vol. 5, 2021

\begin{abstract}
Background
Treatment seeking for people with alcohol use disorders (AUD), injecting drug use and viral hepatitis is low, and has worsened during the coronavirus disease 2019 (COVID-19) pandemic. Deferring clinical care and increasing drug and alcohol use exacerbate the risks of progression for individuals with chronic liver disease from viral hepatitis and/or alcohol-related liver disease (ALD). We explored the impact COVID-19 epidemic may have on patients with chronic liver disease and suggested mechanisms to protect this at-risk population during and after the pandemic.
\end{abstract}

\section{Methods}

We searched PubMed, EMBASE, and Google Scholar for published articles and grey literature and synthesized information about the impact COVID-19 has on patients with chronic liver disease, specifically related to impacts and opportunities to prevent viral hepatitis and ALD.

\section{Results}

The COVID-19 pandemic has caused significant disruption of clinical management of patients with liver disease, but also to alcohol and drug services, needle and syringe programs (NSP), hepatitis B (HBV) screening, and treatment efforts. Social isolation and pandemic-related anxiety have caused an increase in alcohol and drug use. COVID-19 pandemic disruptions may lead to an increase in the incidence of viral hepatitis and ALD, but also cause patients to undertake risky behaviours. The pandemic impacts people with chronic conditions such as HBV to defer medical visits or undertake screening tests. These factors may worsen rates of decompensation for patients with cirrhosis, for whom these deleterious impacts are believed to stay for some time after the pandemic. Interruption in screening, treatment, and monitoring services during the period disrupts chronic disease management of viral hepatitis and ALD and increases the risk of worsening the clinical course of liver disease and delayed diagnosis of hepatocellular carcinoma.

\section{Conclusions}

Successful mitigation of the impact of COVID-19 on the incidence of viral hepatitis and alcoholic liver disease requires adaptive approaches to reach people with injecting drug use and AUD including community-based networks, fixed site and mobile NSP, mail delivery systems, the use of telehealth, and other digital media for promoting and implementing alcohol harm reduction during and after the COVID-19 pandemic.

The coronavirus disease 2019 (COVID-19) pandemic is affecting all the regions of the world. To date, it has resulted in more than 46 million confirmed cases and 1.2 million confirmed deaths globally as at November $1,2020 .{ }^{1}$ Both the number of new infections, cases and deaths continue to rise in many countries throughout the world.

Underlying liver disease is diagnosed in $2 \%-11 \%$ of COVID-19 patients ${ }^{2}$ and the figure could be higher in high hepatitis $\mathrm{B}(\mathrm{HBV})$ and high hepatitis $\mathrm{C}$ virus (HCV) prevalence countries. ${ }^{3}$ In the related severe acute respiratory 
syndrome coronavirus disease-2 (SARS-CoV-2) pandemic, people with viral hepatitis $(\mathrm{VH})$, alcohol-related liver disease (ALD), non-alcoholic fatty-liver disease, liver cirrhosis, and hepatocellular carcinoma (HCC) had a higher risk of getting a severe disease and up to 10 times higher mortality rate $(8.7 \% \text { vs } 0.5 \%)^{4}$ from COVID-19 compared with those without any reported comorbidity. ${ }^{5}$

Patients with substance and alcohol-related problems, in the setting of social isolation, may be less likely to seek help, and as a corollary, may engage in heavier drug and alcohol use. For example, only one in five persons with AUD sought healthcare. ${ }^{6}$

With COVID-19 burden on the health system, chronic liver disease including $\mathrm{VH}$ may be under-screened and under-diagnosed. ${ }^{3}$ To successfully mitigate the challenges from COVID-19, assessment of the impact on this particular population group and designing mechanisms to protect people at-risk of VH and ALD during the time of COVID-19 epidemic and beyond is needed.

Few studies reported the impact COVID-19 epidemic has on liver disease prevention in at-risk populations such as persons with AUD and IDU during and after the pandemic. We explored the impact of COVID-19 on the incidence of and treatment services for $\mathrm{VH}, \mathrm{ALD}$ and presented approaches to protecting the at-risk population from liver disease amid COVID-19 pandemic.

\section{METHODS}

We searched PubMed, EMBASE, and Google Scholar for peer-reviewed articles published between January 1 to November 1, 2020, and grey literature using keywords including "COVID-19”, "SARS-CoV-2", "coronavirus pandemic", "injecting drug use”, "alcohol use disorders", "viral hepatitis", "liver disease". The studies included were review articles, surveys, cohort studies, case-control studies, case series, or evaluation reports. The search terms were combined using the Boolean connectors "AND" and "OR". We performed searches separately for impact of COVId-19 on liver health including 'viral hepatitis prevention AND COVID-19', 'viral hepatitis AND COVID-19 coinfection', 'alcohol use disorders AND COVID-19', 'needle and syringe programs and COVID-19', and 'treatment of viral hepatitis and COVID-19'. All the authors revised the search terms and the first author performed the literature search and screening. The second and last authors revised the relevance of the studies included. We screened and selected articles according to their relevance of the study to each subsection of the review described- articles that reported on the prevalence of AUD, VH during the COVID pandemic, and the progression of liver disease, and treatment of AUD and $\mathrm{VH}$ were retained. We synthesized information about the impact COVID-19 has on the risk of alcohol use disorders and injecting drug use and the implication of these risk behaviours on the incidence and course of $\mathrm{VH}$ and liver disease. We have discussed the strategies to mitigate this impact in four subsections, and included the role of community-based healthcare. We presented the impact and mechanisms to the protection of at-risk population from $\mathrm{VH}$ and ALD in two sections.

\section{RESULTS AND DISCUSSION}

\section{IMPACT OF COVID-19 ON VIRAL HEPATITIS AND LIVER DISEASES}

\section{VIRAL HEPATITIS PREVENTION}

The COVID-19 pandemic has resulted in disruption of health systems in most countries around the world due to the task shifting of health facilities and health personnel to boost the care for COVID-19 patients.

Alcohol and drug services managing drug use and dependence and needle and syringe programs (NSP) have significantly reduced or ceased due to the impact of the pandemic on the health system. A study in England found a $36 \%$ fall in the number of NSP clients and a $29 \%$ reduction in needles distributed. ${ }^{7}$ This exacerbates unsafe injection practices such as needle reuse, loses opportunities for HCV testing and treatment services, and contributes to a silent epidemic of HCV. ${ }^{8}$

\section{RISK TO PATIENTS WITH VIRAL HEPATITIS AND COVID19 COINFECTION}

Patients with COVID-19 exhibit exhaustion of cytotoxic lymphocytes and may be more susceptible to $\mathrm{VH}$ infection. ${ }^{9}$ Routine childhood vaccination services have collapsed in many countries because of the emergency declarations related to COVID-19 epidemic and parents could not bring their children to health facilities for vaccination due to social distancing, restricted travel. This leads to a fall in HBV vaccination coverage leaving millions of children vulnerable to VH. ${ }^{10,11}$ The impact COVID-19 has on the health system also caused a fall in the prevention of vertical transmission of HBV and many children are expected to be born with chronic HBV infection.

\section{REACTIVATION OF VIRAL HEPATITIS}

Hepatitis B reactivation could be a result of SARS-CoV-2 infection or treatments for COVID-19 such as corticosteroids. ${ }^{3}$ A study reported a patient presenting with symptoms of acute hepatitis tested positive for HBsAg, HB$\mathrm{cAb}(\operatorname{IgM})$ and $\mathrm{HBeAb}$, and negative for $\mathrm{HBeAg}$, suggestive of reactivation of an $\mathrm{HBV}$ infection. ${ }^{12}$

\section{INCREASED NEW CASES OF ALCOHOL USE DISORDERS AND ALCOHOL-RELATED LIVER DISEASE}

Alcohol-related liver disease has been on the rise before the COVID-19 crisis. Social isolation and anxiety worsens during such periods causing an increase in alcohol intake, and consequently numbers of people with AUD. ${ }^{6,13}$ Alcohol consumption increases due to many factors including depression, anxiety and stress, loss of job or decline in hours worked, more time staying at home, and boredom compared with pre-pandemic period reports. ${ }^{13}$ These social circumstances are likely to contribute to a rapid rise in AUD relapse and possibly contributes to other problems such as domestic violence. ${ }^{13}$ A survey in Australia reported a $3.5 \%$ increase in high-level alcohol consumption ( $\geqslant 3$ days a week) during May 2020 (COVID-19 epidemic period) compared 
with the $2017 / 18$ report (33.8\% vs $30.3 \%) .{ }^{14}$

\section{AGGRAVATED CLINICAL COURSE OF VIRAL HEPATITIS AND ALCOHOL-RELATED LIVER DISEASE}

\section{SARS-COV-2 -ASSOCIATED LIVER INJURY}

Evidence of liver injury in persons infected with SARSCoV-2 is observed in $14.8 \%-53 \%$ of cases. ${ }^{15}$ Infection with SARS-CoV-2 causes liver injury because angiotensin-converting enzyme 2 , which is a receptor for SARS-CoV-2, is expressed by liver cells. ${ }^{16}$ The SARS-CoV-2-related liver injury occurs due to direct cytotoxicity, immune-mediated severe inflammation, hypoxic hepatitis, reactivation of preexisting liver inflammation, or drug toxicity. ${ }^{17}$

Liver injury from SARS-CoV-2 infection aggravates the course of underlying liver disease such as VH and ALD and the development of decompensated cirrhosis and HCC. ${ }^{18}$ An increase in the frequency of AUD is likely to worsen the progression of hepatitis to liver fibrosis, cirrhosis, or HCC. ${ }^{13}$

\section{DISRUPTION OF CARE AND TREATMENT SERVICES FOR VIRAL HEPATITIS AND LIVER DISEASE}

In many parts of the world, hepatology clinics are converted to emergency service provision units due to a surge in COVID-19 cases. Providers of VH care are often infectious disease specialists but may be hepatologists repurposed to engage in the treatment of COVID-19 patients, thus bringing hepatology clinics to closure. Liver disease patients may therefore experience difficulty to be reassessed for initiating antiviral treatment or collecting scripts for ongoing treatment. This increases the risk of liver injury and decompensation from immune active or immune escape HBV, particularly with treatment discontinuation for patients already identified as needing treatment for viral suppression. ${ }^{19,20}$

Patients with HBV are recommended to undergo agebased screening to improve early detection of hepatocellular carcinoma (HCC) and consequently improve survival. Interruption of normal clinical services for HBV patients impacts HCC screening ultra-sonography, image interpretation and further investigation of lesions, and if necessary appropriate treatment. In this way COVID-19 impacts HBV patients in a cascade of HBV treatment and assessment but also surveillance for HCC complications. The disruption of these services will have further knock-on effects for liver patients. Even with services available, patients may be too scared to attend for planned investigations, avoiding hospitals and health care and isolating in the community.

Patients with $\mathrm{VH}$ and liver disease are at a higher risk of getting severe COVID-19.21 The perception of being at a higher risk of dying from COVID-19, coupled with travel restrictions, contributes to $\mathrm{VH}$ patients staying at home and refrain from collecting scripts or visiting follow-up clinics. COVID-19 complicated the existing liver disease care issues in low and middle-income countries including exacerbating the already high rates of undiagnosed disease, discontinuation of follow-up and treatment in identified patients. ${ }^{22}$ In many low-and middle-income countries, health facilities are usually stocked out of antiviral drugs including direct- ing-acting antivirals for HCV and anti-HBV drugs due to procurement inaccessibility and financial constraints ${ }^{23}$ and gets worse during such a pandemic. Existing inequalities in healthcare particularly in rural areas of low and middleincome countries will be worsened by the COVID-19 pandemic due to travel restrictions and movement to urban areas to generate income for livelihood of many families, making the existing out-of-pocket expenditure for healthcare a more impossible dream. ${ }^{24}$

Disruption of addiction medicine and alcohol harm reduction services, such as education and AUD treatment will also be impacted by COVID-19, potentially contributing to an increased number of alcohol-related liver fibrosis and cirrhosis cases. ${ }^{25}$ Liver transplantation may be impacted, with challenges to donor screening for the suitability of organs is challenging in the presence of SARS-COV-2 pandemic. Transplantation from SARS-COV-2 exposed donors is not recommended due to the paucity of evidence about the safety of organs in this donor group. ${ }^{26}$ Figure 1 presents the detailed impact of the COVID-19 on viral hepatitis, liver disease and decompensation of cirrhosis.

\section{HOW CAN WE PROTECT AT-RISK PERSONS FROM VIRAL HEPATITIS AND LIVER DISEASE?}

Existing liver disease care systems faced the care for liver diseases with fewer staff, testing and diagnostic facilities, and in some cases, converted to COVID-19 treatment centres. In low- and middle-income countries, the already existing pressure on the healthcare system has been disrupted by the pandemic. ${ }^{7,24}$ Viral hepatitis and ALD prevention and treatment strategies adapted to the situation of the ongoing pandemic are needed.

\section{TREATING ALCOHOL USE DISORDER AND PREVENTING ALCOHOL-RELATED LIVER DISEASE}

People more likely to develop AUD from the circumstances associated with COVID-19 pandemic, and persons with AUD are at high risk for ALD and are a neglected yet priority group for prevention. ${ }^{27}$ Studies found only one in five persons with AUD reach out to health facilities ${ }^{6}$ and this may be expected to be lower during the pandemic. Addiction treatment providers and hepatologists need to actively reach out and engage at-risk populations, to treat AUD with counselling services and pharmacotherapy where appropriate.

Internet-based counselling and education services can be used to reach people at risk of developing AUD. For people with AUD before the COVID-19 pandemic, alcohol harm reduction services can be re-energised to ensure the continuation of addiction treatment, monitor AUD relapse, or conduct surveillance for symptoms of decompensation in those with liver disease who relapse. ${ }^{13}$ Telehealth and text messaging services can be used to improve engagement and as a bridge to deliver addiction treatment and counselling about alcohol abstention benefits for people at risk of liver disease from AUD. Digital media such as television and social media networks are essential tools to deliver health information about alcohol abstinence and alternative leisure activities such as physical exercise. 


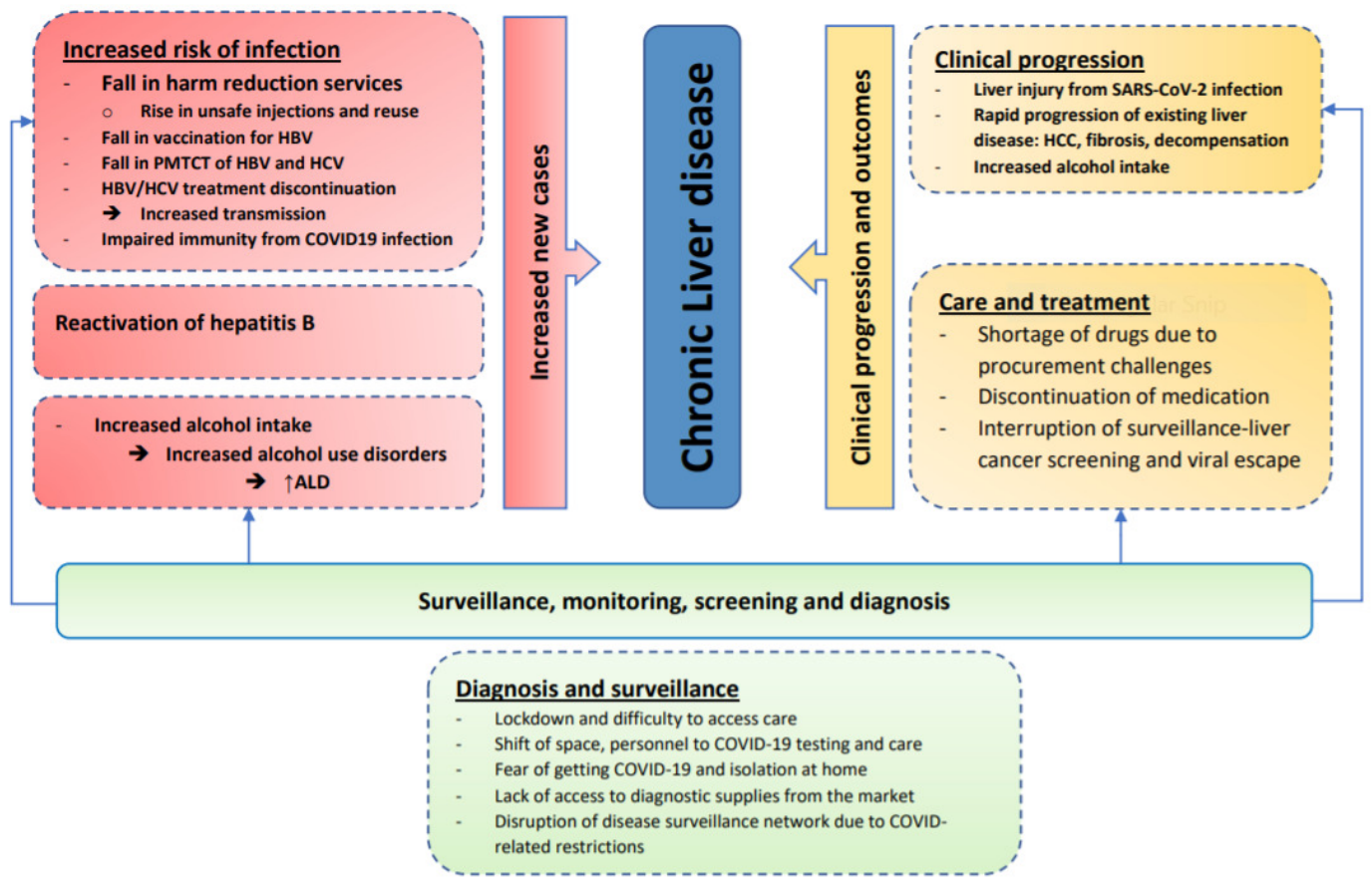

\section{Figure 1. Impact of COVID-19 epidemic on incidence of viral hepatitis and alcohol-related liver disease, and clinical progression.}

COVID-19-related social restriction and interruption of clinical care and reduce uptake increases chronic liver disease in three ways: increased new infections and cases of alcohol-related liver disease (red boxes), clinical progression to advanced liver disease of existing disease(yellow boxes), and lack of surveillance and screening (green boxes). ALD, Alcohol-related liver disease; COVID-19, Coronavirus disease 2019; HBV, hepatitis B virus; HCC, hepatocellular carcinoma; HCV, hepatitis C virus; PMTCT, prevention of mother to child transmission; SARS-CoV-2; severe acute respiratory syndrome coronavirus 2

\section{MAINTAINING HARM REDUCTION AND NEEDLE AND SYRINGE SERVICES}

Social distancing measures will remain in place for some time creating a 'new normal' and operational change to NSP are needed. Improving awareness of the presence of telehealth services and modified approaches to deliver NSP is important to retain patients, ensure both safe injection, and reduce COVID-19 transmission risk. ${ }^{7}$ Delivery by post, home delivery and peer-based distribution while keeping proper social distancing will be useful to sustain supplies for safe injection. ${ }^{7}$

Needle and syringe services and addiction treatment units can be designated as essential services by health departments, which ensures the venues still being open, staff in place for consultation, and enough budget available to maintain supplies. HCV testing is expected to have ceased in many NSPs. Reinitiating rapid testing and treatment is useful to prevent a rise in HCV incidence. ${ }^{8}$

Planning for and delivering extra supplies of opiate replacement therapy such as methadone and buprenorphine for substance use disorders may help to mitigate the impact of COVID-19 on treatment continuation. Healthcare providers can offer extra syringes and other harm reduction supplies (such as gloves, and hand sanitizers) for safe injecting drug use to ensure a continued supply. Ensuring sufficient capacity to prescribe direct-acting antiviral therapy for HCV and methadone/buprenorphine for opioid dependence are critical to prevent relapse of these disease conditions and prevent liver disease. ${ }^{28}$ Telehealth can be used to engage, monitor adherence and clinical progress for those with viral hepatitis.

\section{MAINTAIN ROUTINE VACCINATION AND PREVENT MOTHER-TO-CHILD TRANSMISSION OF HEPATITIS B}

Due to the possibility of an outbreak that could further disrupt the health system or high mortality from vaccine-preventable diseases, countries shall label routine immunization services as a priority health service. ${ }^{29}$ Education to families and communities about the importance of completing the HBV vaccination is vital. For families with eligible children, making calls and sending reminder texts are helpful to keep HBV vaccination coverage. ${ }^{11}$ There is a significant drop in HBV vaccination because of the disruption from COVID-19 epidemic in many countries, a rapid vaccination to ensure catch-up vaccination is needed for children who missed doses.

Hepatitis B positive pregnant women should be educated to on the importance of preventive care for their infant (HBV immunoglobulin and birth dose and continued HBV vaccinations). In the case of unplanned delivery, supplying documentation will be useful to provide to the delivery staff to help them with the care. Hepatitis B immunoglobulin and $\mathrm{HBV}$ vaccine are provided within 12 hours of delivery when possible. ${ }^{30}$

\section{PREVENTING LIVER DECOMPENSATION}

Because of the immunocompromised status of persons with liver disease, secondary infections, hepatic encephalopathy, 
and upper gastrointestinal bleeding are expected to increase and intense surveillance and treatment are needed for these patients. ${ }^{20}$ Viral hepatitis and liver disease patients are treated according to the pre-existing guidelines for the treatment of these conditions, with some exceptions in SARS-CoV-2 coinfection. For persons with HBV infection, antiviral treatment will be delayed unless a flare is suspected. ${ }^{31}$ Treatment of HCC patients with COVID-19 is recommended to be delayed. ${ }^{19}$ Given $\mathrm{VH}$ and liver disease patients are categorised as a high-risk group for developing a severe disease if they contract SARS-CoV-2, priority must balance the prevention of COVID-19 infection with the progression of liver disease. ${ }^{31}$

To achieve this, tailored monitoring of treatment and clinical progress in persons with $\mathrm{VH}$ and liver disease using telemedicine services may reduce the risk of exposure to COVID-19 cases and health care workers to SARS-CoV-2. It is also an efficient method of health service delivery as it saves the use of personal protective equipment, visiting time, and can identify patients who need hospitalization. ${ }^{32}$ Multi-month dispensing is a particularly suitable alternative to alleviate the challenges of antiviral medication stockout.

\section{ROLE OF COMMUNITY-BASED HEALTHCARE FOR MITIGATION}

The community-based health workforce has strong ties with community actors and had significant success stories in disease prevention, particularly in low-and middle-income countries. Ensuring community health care workers are educated and trained to identify, assess and refer patients with liver disease or substance use problems, will lessen the compromise on COVID-19 health responses. 33 The use of community-based health workforce in collaboration with addiction treatment and hepatology specialty services are essential to creating an accessible and resilient harm reduction and NSP service. Existing community-based primary health care offers a cost-effective and culturally appropriate service provision for the prevention of $\mathrm{VH}$ and ALD in hardto-reach populations. ${ }^{34}$

\section{CONCLUSIONS}

The COVID-19 pandemic, directly and indirectly, threatens the lives of patients with viral hepatitis, liver disease and substance use disorders. The impact on hepatitis clinics and addiction medicine units leaves patients at risk of deterioration from their underlying disease, as well as screening measures to identify complications (such as HCC in VH patients) or relapse in patients with IDU and alcohol dependence. Because of the social distancing and travel restriction measures, social isolation and stress appear anecdotally to have taken a significant toll on patients with substance- and alcohol-use disorder, significantly increas- ing the risk of relapse. With task-shifting to deal with the COVID-19 epidemic, clinical care for $\mathrm{VH}$ and liver disease have been impaired, and this impact may be most be pronounced for patients with liver cirrhosis and HCC patients, who are among the most vulnerable in the community. Interruption of harm reduction and NSP activities and routine HBV vaccination will have impacts on horizontal transmission of HBV and HCV, and prevention of mother to child vertical transmission of $\mathrm{HBV}$, increasing the risk of a rise in the incidence of $\mathrm{VH}$.

There remain opportunities to help our patients with liver disease and substance use disorders. Upskilling community-based healthcare workers and infrastructure to reduce the need for patients to attend hospital offers a pathway to improve care and reduce their risk of COVID19 transmission while keeping them engaged. Using disruptive technology, optimising telehealth, and internet-based education and other digital media to engage, educate and treat patients with these diseases may help to reduce the harm imposed by COVID-19 to individuals and the health system, and provide a sustainable pathway through and beyond the pandemic era.

\section{FUNDING}

None

\section{AUTHORSHIP CONTRIBUTIONS}

BT was involved in the inception, design, literature search, drafted the manuscript and revised different versions. PC and PV were involved in the inception, design, write up of manuscript, and revised the manuscript. All the authors read and approved the final version of the manuscript.

\section{COMPETING INTERESTS}

The authors completed the Unified Competing Interest form at www.icmje.org/coi_disclosure.pdf (available upon request from the corresponding author) and declare no conflicts of interest.

\section{CORRESPONDENCE TO:}

\section{Belaynew W Taye (MD, MPH)}

Bahir Dar University, Department of Epidemiology

Bahir Dar Ethiopia and

The University of Queensland, Brisbane Australia bewassie@gmail.com

Submitted: November 27, 2020 GMT, Accepted: February 08, 2021 GMT 


\section{REFERENCES}

1. World Health Organization (WHO). COVID-19 Weekly Epidemiological Update-3 November 2020. World Health Organization; 2020. Accessed November 6, 2020. https://www.who.int/publications/m/item/we ekly-epidemiological-update---3-november-2020

2. Jothimani D, Venugopal R, Abedin MF, Kaliamoorthy I, Rela M. COVID-19 and the liver. Journal of hepatology. Published online 2020:S0168-8278(20)30377-9. doi:10.1016/j.jhep.202 $\underline{0.06 .006}$

3. Reddy KR. SARS-CoV-2 and the Liver: Considerations in Hepatitis B and Hepatitis C Infections. Clinical Liver Disease. 2020;15(5):191-194. doi:10.1002/cld.970

4. Moon AM, Webb GJ, Aloman C, et al. High mortality rates for SARS-CoV-2 infection in patients with pre-existing chronic liver disease and cirrhosis: Preliminary results from an international registry. Journal of Hepatology. Published online May 21, 2020. doi:10.1016/i.jhep.2020.05.013

5. Yang R, Gui X, Zhang Y, Xiong Y. The role of essential organ-based comorbidities in the prognosis of COVID-19 infection patients. Expert Review of Respiratory Medicine. Published online 2020:1-4. doi:1 $\underline{0.1080 / 17476348.2020 .1761791}$

6. Finlay I, Gilmore I. Covid-19 and alcohol-a dangerous cocktail. BMJ. 2020;369:m1987. doi:10.113 6/bmi.m1987

7. Whitfield M, Reed H, Webster J, Hope V. The impact of COVID-19 restrictions on needle and syringe programme provision and coverage in England. Int $J$ Drug Policy. Published online 2020:102851. doi:10.10 16/j.drugpo.2020.102851

8. Bartholomew TS, Nakamura N, Metsch LR, Tookes HE. Syringe services program (SSP) operational changes during the COVID-19 global outbreak. International Journal of Drug Policy. Published online June 12, 2020:102821. doi:10.1016/j.drugpo.2020.102 $\underline{821}$

9. Zheng M, Gao Y, Wang G, et al. Functional exhaustion of antiviral lymphocytes in COVID-19 patients. Cell Mol Immunol. 2020;17(5):533-535. doi:1 $\underline{0.1038 / s 41423-020-0402-2}$

10. Santoli JM. Effects of the COVID-19 pandemic on routine pediatric vaccine ordering and administration-United States, 2020. MMWR Morbidity and Mortality Weekly Report. 2020;69.
11. Bramer CA, Kimmins LM, Swanson R, et al. Decline in child vaccination coverage during the COVID-19 pandemic - Michigan Care Improvement Registry, May 2016-May 2020. MMWR Morbidity and mortality weekly report. 2020;68(22).

12. Aldhaleei WA, Alnuaimi A, Bhagavathula AS. COVID-19 Induced Hepatitis B Virus Reactivation: A Novel Case From the United Arab Emirates. Cureus. 2020;12(6):e8645-e8645. doi:10.7759/cureus.8645

13. Da BL, Im GY, Schiano TD. COVID-19 Hangover: A Rising Tide of Alcohol Use Disorder and AlcoholAssociated Liver Disease. Hepatology. Published online May 5, 2020. doi:10.1002/hep.31307

14. Nicholas B, Ben E, Matthew G, Kate S. Alcohol consumption during the COVID-19 period: May 2020. ANU Centre for Social Research and Methods; 2020.

15. Wang Y, Liu S, Liu H, et al. SARS-CoV-2 infection of the liver directly contributes to hepatic impairment in patients with COVID-19. J Hepatol. Published online May 10, 2020. doi:10.1016/i.jhep.2020.05.002

16. Xu L, Liu J, Lu M, Yang D, Zheng X. Liver injury during highly pathogenic human coronavirus infections. Liver International. 2020;40(5):998-1004. d oi:10.1111/liv.14435

17. Sun J, Aghemo A, Forner A, Valenti L. COVID-19 and liver disease. Liver Int. 2020;40(6):1278-1281. do i:10.1111/liv.14470

18. Qi X, Wang J, Li X, et al. Clinical course of COVID-19 in patients with pre-existing decompensated cirrhosis: initial report from China. Hepatol Int. Published online May 22, 2020:1-5. doi:1 0.1007/s12072-020-10051-z

19. Boettler T, Newsome PN, Mondelli MU, et al. Care of patients with liver disease during the COVID-19 pandemic: EASL-ESCMID position paper. JHEP Reports. 2020;2(3):100113. doi:10.1016/j.jhepr.2020.1 $\underline{00113}$

20. Zhang C, Shi L, Wang FS. Liver injury in COVID-19: management and challenges. The Lancet Gastroenterology \& Hepatology. 2020;5(5):428-430. do $\mathrm{i}: 10.1016 / \mathrm{s} 2468-1253(20) 30057-1$

21. US Centers for Disease Prevention and Control. Coronavirus disease 2019 (covid 19). People Who Are at Increased Risk for Severe Illness. Accessed July 18, 2020. https://www.cdc.gov/coronavirus/2019-ncov/ne ed-extra-precautions/people-at-increased-risk.html 
22. Gupta N, Desalegn H, Ocama P, et al. Converging pandemics: implications of COVID-19 for the viral hepatitis response in sub-Saharan Africa. Lancet Gastroenterol Hepatol. 2020;5(7):634-636. doi:10.101 6/S2468-1253(20)30155-2

23. Taye BW. A Path to Ending Hepatitis C in Ethiopia: A Phased Public Health Approach to Achieve Micro-Elimination. Am J Trop Med Hyg. 2019;101(5):963-972. doi:10.4269/aitmh.19-0295

24. Pley CM, McNaughton AL, Matthews PC, Lourenço J. The global impact of the COVID-19 pandemic on the prevention, diagnosis and treatment of hepatitis B virus (HBV) infection. BMJ Global Health. 2021;6(1):e004275. doi:10.1136/bmigh-202 $\underline{0-004275}$

25. Karimi-Sari H, Rezaee-Zavareh MS. COVID-19 and viral hepatitis elimination programs: Are we stepping backward? Liver Int. Published online April 22, 2020. doi:10.1111/liv.14486

26. Aslam S, Mehra MR. COVID-19: Yet another coronavirus challenge in transplantation. J Heart Lung Transplant. 2020;39(5):408-409. doi:10.1016/i.healu $\underline{\text { n.2020.03.007 }}$

27. Mota P. Avoiding a new epidemic during a pandemic: The importance of assessing the risk of substance use disorders in the COVID-19 era. Psychiatry Res. 2020;290:113142-113142. doi:10.1016/ j.psychres.2020.113142

28. Harm reduction coalition. Syringe Services and Harm Reduction Provider Operations During the COVID-19 Outbreak. Accessed July 15, 2020. https://h armreduction.org/wp-content/uploads/2020/03/COVI D19-harm-reduction-providers-1.pdf
29. World Health Organization (WHO). Guiding principles for immunization activities during the COVID-19 pandemic: interim guidance. https://ww w.who.int/publications/i/item/guiding-principles-forimmunization-activities-during-the-covid-19-pande mic-interim-guidance

30. Centers for Disease prevention and Control (CDC). Interim guidance to prevent mother-to-child transmission of hepatitis B virus during COVID-19-related disruptions in routine preventive services. https://www.hhs.gov/hepatitis/blog/2020/0 5/01/cdc-mother-child-hbv-transmission-preventioncovid-guidance.html

31. George L, Ward JW. Synthesis of Liver Associations Recommendations for Hepatology and Liver Transplant Care During the COVID-19 Pandemic. Clinical Liver Disease. 2020;15(5).

32. Fix OK, Serper M. Telemedicine and Telehepatology During the COVID-19 Pandemic. Clinical Liver Disease. 2020;15(5):187-190. doi:10.100 2/cld.971

33. World Health Organization and the United Nations Children's Fund (UNICEF). Community-based health care, including outreach and campaigns, in the context of the COVID-19 pandemic. Published online 2020 .

34. Centers for Disease Prevention and Control (CDC). Framework for Implementation of COVID-19 Community Mitigation Measures for Lower-Resource Countries. Published online June 2020. https://www.c dc.gov/coronavirus/2019-ncov/global-covid-19/comm unity-mitigation-measures.html 